\title{
150 ans de croissance urbaine
}

Madame France Guérin-Pace, Madame Denise Pumain

\section{Citer ce document / Cite this document :}

Guérin-Pace France, Pumain Denise. 150 ans de croissance urbaine. In: Economie et statistique, $\mathrm{n}^{\circ} 230, \mathrm{Mars}^{1990 .}$ Communes, métropoles, régions : l'espace français. pp. 5-16;

http://www.persee.fr/doc/estat_0336-1454_1990_num_230_1_5426

Document généré le 23/05/2016 


\begin{abstract}
150 Years of Urban Growth - After a century and a half of gradual urbanization, the number of French cities has increased by a factor of three, and that of urban residents by a factor of seven. Three French people out of four now live in a city and, among them, three out of five live in a city of over a hundred thousand inhabitants.

This urbanization was not uniform when examined by region or city. Some urban units developed extensively, others declined noticeably. Most of them did not go through an even process of evolution. Hence, in the classification of cities according to size, the changes in rank were frequent and sometimes considerable. However, rather homogeneous evolution profiles can be distinguished, which are regionally well-defined, and the shape of the pyramird by size has remained more or less unchanged. In the long term, the only notable change is a degree of decentralization: middle-sized cities are becoming larger. But the overall structure of the hierarchy is very stable over time, with Paris remaining in a highly dominant position. The permanence of this pyramid is compatible with the random growth of cities.
\end{abstract}

\title{
Résumé
}

Après un siècle et demi d'urbanisation progressive, la France compte trois fois plus de villes et sept fois plus de citadins. Trois français sur quatre sont désormais urbains et parmi eux, trois sur cinq habitent une ville de plus de 100.000 habitants. Cette urbanisation n'a pas été uniforme selon les régions ou les villes. Certaines unités urbaines ont connu une croissance forte, d'autres un net déclin, la plupart n'ont pas suivi une trajectoire régulière. Dès lors, dans le classement des villes selon leur taille, les changements de rang ont été multiples et parfois très importants. Pourtant, on distingue des profils d'évolution assez homogènes, régionalement bien délimités, et l'allure de la pyramide des tailles est à peu près restée la même. Sur le long terme, le seul changement notable est une certaine déconcentration : le poids des villes moyennes se renforce. Mais la forme générale de la hiérarchie est très stable dans le temps, avec notamment une forte primauté parisienne. La permanence de cette pyramide est compatible avec une croissance aléatoire des villes.

\section{Resumen}

150 años crecimiento urbano - Luego de un siglo y medio de urbanization progresiva Francia cuenta con una proporción de ciudades très veces mayor y con un número de habitantes urbanos siete veces más importante. De cada cuatro franceses, tres son urbanos y dentro de ese porcentaje, tres de cada cinco viven en una ciudad de mas de 100.000 habitantes.

Esta urbanization no ha sido uniforme según las regiones o las ciudades. Ciertas ciudades urbanas experimentan un fuerte crecimiento, otras, en cambio, un neto retroceso. La mayoría de ellas no han seguido una trayectoria regular. Por lo tanto, en la clasificación de las ciudades según su dimension, los cambios de position fueron multiples y en muchas ocasiones muy importantes. Sin embargo, se pueden distinguir perfiles de evolución bastante homogéneos, bien limitados regionalmente y el aspecto de la pirámide que refleja las dimensiones se mantuvo sin mayores cambios. A largo plazo, la única transformation notable es la de una deconcentración, por ende, el peso de las ciudades medianas se refuerza. No obstante, el contorno global de la jerarquía sigue siendo muy estable en el tiempo y en especial, con una fuerte primacía de París.

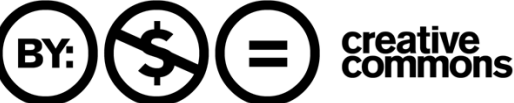




\title{
150 ans de croissance urbaine
}

France

Guérin-Pace, Denise Pumain*
* France Guérin-Pace est allocataire de recherche au CNRS, Equipe P.A.R.I.S et Denise Pumain est Professeur à l'Université Paris I et conseiller technique à I'INED.

Benoit Riandey et Arnaud Bringé omt par licipé à la réalisation da fichicr des unite's urbaines.

Les nombres entre (rochets remoiemt à la bibliographice 'n fin d'article.

\begin{abstract}
Après un siècle et demi d'urbanisation progressive, la France compte trois fois plus de villes et sept fois plus de citadins. Trois Français sur quatre sont désormais urbains et parmi eux, trois sur cinq habitent une ville de plus de 100.000 habitants.
\end{abstract}

Cette urbanisation n'a pas été uniforme selon les régions ou les villes. Certaines unités urbaines ont connu une croissance forte, d'autres un net déclin. La plupart n'ont pas suivi une trajectoire régulière. Dès lors, dans le classement des villes selon leur taille, les changements de rang ont été multiples et parfois très importants.

Pourtant, on distingue des profils d'évolution assez homogènes et chaque type de croissance est représenté dans la plupart des régions. L'allure de la pyramide des tailles est à peu près restée la même. Sur le long terme, le seul changement notable est un renforcement du poids des grandes villes. Mais la forme générale de la hiérarchie est très stable dans le temps, avec notamment une forte primauté parisienne. La permanence de cette pyramide est compatible avec une croissance aléatoire des villes.

Autant le processus d'urbanisation considéré sur le long terme semble cohérent et régulier, autant la croissance urbaine observée ville par ville et sur de courts intervalles de temps apparaît capricieuse. fluctuante, et imprévisible. Les multiples variations locales de la population des villes, apparemment aléatoires, sont compatibles avec la très grande stabilité de la trame des villes et de la forme de la hiérarchie urbaine. Ces fluctuations reflètent les interdépendances et les relations de compétition et de concurrence entre les villes. Elles contribuent à assurer la permanence de l'organisation hiérarchique et spatiale de ce qui constitue un véritable système de villes. Ce système maintient ses propriétés essentielles au cours du temps, par-delà les transformations profondes des activités et des populations urbaines. Il subit cependant certaines altérations, du fait de son inscription dans un espace où les "distances-temps" se sont considérablement réduites depuis un siècle et demi.

\section{Une urbanisation lente et tardive}

Au cours du siècle et demi écoulé, la France qui était un pays à $80 \%$ rural est devenu aux trois quarts urbanisé. La population urbaine, mais aussi le nombre des villes et leur taille moyenne ont augmenté (sur les sources et les définitions, voir l'encadré page suivante). 


\section{LE FICHIER : 150 ANS D'HISTOIRE DES VILLES}

L'histoire de l'urbanisation de la France semble aujourd'hui bien connue. La représentation que l'on en a relève surtout de deux types d'études statistiques : celles qui ont analysé globalement l'évolution de la population urbaine, au mieux par régions, ou par classes de taille de communes, et celles qui ont décrit la dynamique d'une ville ou du réseau des villes d'une région, le plus souvent selon une approche monographique. Beaucoup plus rares sont les études, dites "désagrégées", qui analysent l'évolution des populations urbaines ville par ville, simultanément pour l'ensemble du territoire, et pour une très longue période (Pumain, [6], GuérinPace, [4]).

Cette lacune s'explique en partie par l'ampleur de l'information à rassembler : dès le début du XIXe siècle, les dénombrements font apparaître plusieurs centaines de communes urbaines, ily en a plus de 4000 aujourd'hui. En outre, pour obtenir des unités urbaines qui soient comparables dans le temps et pertinentes pour l'analyse de l'urbanisation, il faut agréger les communes urbanisées en continuité, en s'inspirant du modèle que I'INSEE a proposé à partir de 1954 pour la définition d'agglomérations urbaines multicommunales. Le fichier "Urbanisation de la France" réalisé en 1985 par I'INED propose une reconstitution de la population des agglomérations urbaines à tous les recensements compris entre 1831 et 1982 ([7] et [8]).

Les principales informations contenues dans ce fichier sont, outre la population à chacun des 27 recensements, l'identification de l'unité urbaine, ses coordonnées géographiques, son année d'apparition, son année de dernière disparition éventuelle et les différents "accidents" pouvant survenir : absorption, retour au rural provisoire ou définitif. Ces types d' "avatars" sont dénombrés dans le tableau 1. La carte 1 précise la localisation de toutes les unités urbaines (2388) répertoriées dans le fichier.

Mis à part ce fichier, il n'existe jusqu'à présent en France aucune série de données statistiques homogènes décrivant le phénomène d'urbanisation sur une longue période. Pour le réaliser, il a fallu dans une première étape rechercher les statistiques de la population données par communes et publiées au Bulletin des Lois entre 1821 et 1876 et en volumes de Dénombrements après cette date. Ces informations n'existaient dans une version informatisée, dans la Base de Données Communales de I'INSEE, que pour la période 1954-1982. Ainsi, dans un premier temps, I'INED a constitué un fichier informatisé de toutes les communes, dont la population a dépassé 2000 habitants à l'un des recensements compris entre 1831 et 1982, dit "fichier des communes urbaines".

Afin d'assurer une cohérence chronologique des définitions du caractère urbain il a été nécessaire d'effectuer, à partir du dénombrement des communes urbaines, une reconstitution des unités urbaines ou agglo. mérations de 1831 à 1982, en adoptant une délimitation variable. Pour simplifier cette reconstitution, un seul critère d'annexion des communes à une ville-centre a été retenu: ont été incluses dans l'agglomération, à chaque date, les communes contiguës dont la population dépassait 2000 habitants, à condition que ces communes fassent partie de l'agglomération définie par l'INSEE en 1954. Le fichier des unités urbaines a été élaboré en deux temps, une première partie couvrant la période 1831-1911 a été mise au point par D. Pumain dès 1978 et la seconde, de 1911 à 1982, parl'INED en 1984. Alussi, il existe entre les deux parties du fichier quelques différences quant aux définitions de la population et au choix du seuil urbain.

Tableau 1

\begin{tabular}{|c|c|c|c|c|c|c|}
\hline \multirow{2}{*}{ Recensements } & \multicolumn{5}{|c|}{ Nombre d'unités urbaines } & \multirow{2}{*}{$\begin{array}{c}\text { Nombre de } \\
\text { communes } \\
\text { urbaines }\end{array}$} \\
\hline & Création & Absorption & Disparition & Réurbanisation & Total & \\
\hline 1831 & 47 & 1 & 12 & - & 719 & 782 \\
\hline 1836 & 34 & 1 & 5 & 4 & 751 & 824 \\
\hline 1841 & 38 & 2 & 8 & 3 & 782 & 873 \\
\hline 1846 & 43 & 1 & 7 & 2 & 819 & 917 \\
\hline 1851 & 33 & 0 & 17 & 4 & 839 & 947 \\
\hline 1856 & 45 & 2 & 4 & 7 & 885 & 1012 \\
\hline 1861 & 43 & 0 & 6 & 4 & 926 & 1066 \\
\hline 1866 & 23 & 4 & 19 & 1 & 927 & 1085 \\
\hline 1872 & 26 & 1 & 13 & 4 & 943 & 1112 \\
\hline 1876 & 29 & 3 & 14 & 11 & 966 & 1162 \\
\hline 1881 & 13 & 2 & 15 & 2 & 964 & 1180 \\
\hline 1886 & 26 & 0 & 20 & 5 & 975 & 1201 \\
\hline 1891 & 24 & 1 & 20 & 8 & 986 & 1235 \\
\hline 1896 & 27 & 7 & 9 & 11 & 1008 & 1295 \\
\hline 1901 & 22 & 7 & 22 & 3 & 1004 & 1331 \\
\hline 1906 & 36 & 4 & 29 & 3 & 1010 & 1367 \\
\hline 1911 & 155 & 10 & 36 & 33 & 1152 & 1583 \\
\hline 1921 & 47 & 3 & 144 & 4 & 1056 & 1522 \\
\hline 1926 & 49 & 3 & 33 & 55 & 1124 & 1671 \\
\hline 1931 & 40 & 1 & 35 & 20 & 1148 & 1734 \\
\hline 1936 & 23 & 0 & 36 & 23 & 1158 & 1751 \\
\hline 1946 & 60 & 2 & 68 & 31 & 1179 & 1802 \\
\hline 1954 & 30 & 11 & 43 & 35 & 1190 & 2011 \\
\hline 1962 & 175 & $\theta$ & 22 & 67 & 1401 & 2518 \\
\hline 1968 & 251 & 168 & 11 & 47 & 1520 & 4039 \\
\hline 1975 & 173 & 49 & 21 & 19 & 1642 & 4475 \\
\hline 1982 & 191 & 43 & 27 & 18 & 1781 & 4905 \\
\hline
\end{tabular}




\section{Definition de la population}

Pour la première partie du fichier (1831-1911), la population considérée est la population totale : elle comprend des doubles comptes, c'està-dire qu'elle recense certaines personnes à la fois dans des établissements situés dans cette commune et dans leur commune de résidence habituelle (par exemple les militaires, pensionnaires, détenus, etc.). II faut signaler par ailleurs que, pour des raisons matérielles, ces données de population ont été arrondies, selon le cas, à la centaine supérieure ou inférieure. Entre 1911 et 1982, on considère la population municipale, c'est à dire essentiellement les personnes qui occupent des logements d'habitation dans la commune. Cette population se décompose elle-méme en deux parties: la population "agglomerée au chef-lieu" et la population éparse. Cette population est donnée à l'unité près.

\section{Choix du seuil de l'urbain}

L'INSEE a adopté pour la définition des communes urbaines un seuil de 2000 habitants agglomérés, mais de nombreux historiens en récusent la validité. Ainsi, Dupeux [9] établit à 3000 habitants agglomérés au cheflieu le seuil minimal urbain. Le seuil retenu par D. Pumain est intermédiaire entre celui de I'INSEE et celui de Dupeux, à 2500 habitants agglomérés au cheflieu. A partir de 1911 et jusqu'en 1982, est utilisé pour la constitution du fichier le seuil de 2000 habitants defini par I'INSEE, moins restrictif que le précédent.

Délimitation des agglomérations ou unités urbaines Jusqu'en 1954, le critère d'agrégation des communes est le suivant: si deux ou plusieurs communes déjà urbaines sont contiguës, alors elles constituent une méme agglomération. A partir de 1954, sont appliquées les différentes définitions de l'INSEE, la première valable jusqu'en 1962 et la seconde jusqu'à nos jours. Ces

Carte 1 différentes définitions créent un problème de continuité et d'utilisation des données. II a donc été nécessaire d'enregistrer deux fois l'information pour les recensements coïncidant avec des changements de définition. Ainsi deux recensements sont doublés dans le fichier et appelés, pour plus de commodité, 1911 et 1912 puis 1954 et 1955. La modification du seuil de l'urbain à partir de 1911 crée dans la série, dite de 1912, 155 unités urbaines supplémentaires, mais l'utilisation de l'une ou de l'autre de ces deux séries pour l'étude de l'évolution urbaine n'entraîne pas de modification importante. Le passage de la définition de 1954 à celle de 1962 s'avère plus délicat. En effet, lors du recensement de 1954 certaines communes urbaines contiguës mais dépendant de pôles d'attraction différents étaient dissociées. II s'ensuit que 230 unités urbaines, qui étaient considérées comme indépendantes dans ce recensement, furent aussitót annexées en 1962 à l'unité urbaine voisine. La série, dite de 1955, reconstitue pour 1954 des agglomérations dont la délimitation correspond aux criteres de l'ensemble du fichier.

\section{Qualité des données}

Chassagne [10] signale des surestimations systématiques des populations de Lyon ou Marseille à la fin du $19 e \dot{m} e$ siécle, qui espéraient chacune devenir la seconde ville de France. Le recensement de 1841 comporte aussi des erreurs. Pour établir l'assiette de l'impót, on avait voulu recenser la population de fait et non la population domiciliée seulement, comme cela s'était pratiqué jusque-là. Ainsi, les populations recensées en 1841 sont sous-estimées pour une majorité d'unités urbaines, et plus systématiquement dans les grandes villes que dans les petites. Ce biais se ressent dans le calcul des taux de croissance de la population des unités urbaines. Ceux-ci sont donc sous-estimés pour la période 1836-1841 et surestimés entre 1841 et 1846.

Que sont devenues les unités urbaines (1831-1982) ?

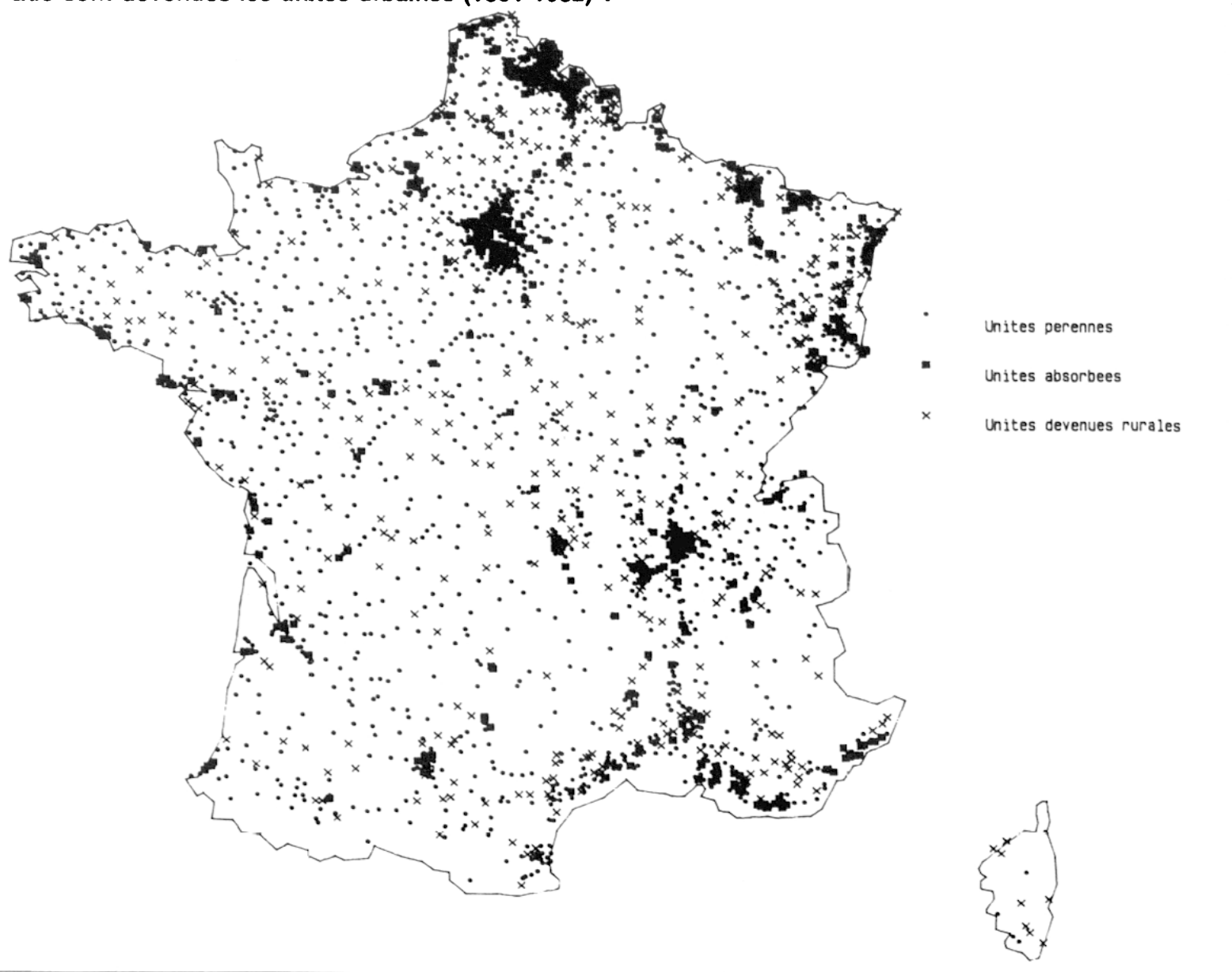




\section{Graphique I \\ Plus de villes, encore plus d'urbains}

\section{Graphique II Les villes se rapprochent}

* Distance moyenne des villes à leur plus proche voisine de même taille.

\section{Graphique III}

Une croissance fluctuante

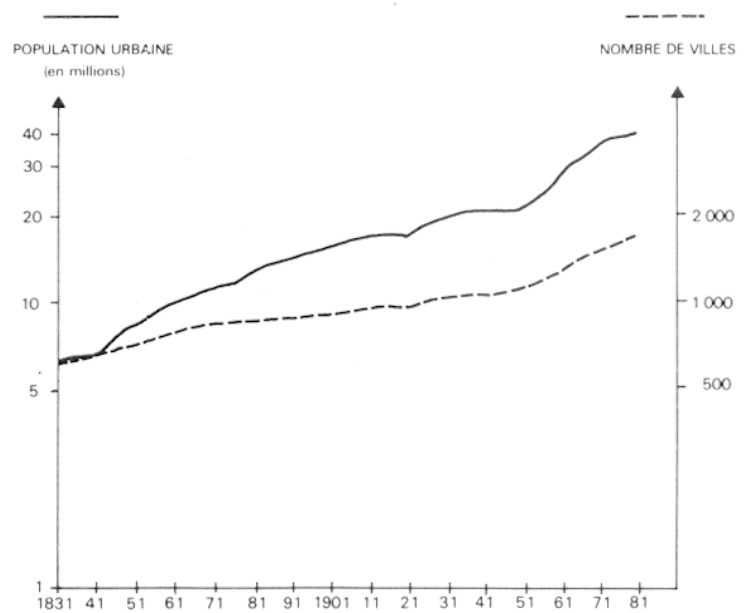

\section{II-A - Les grandes villes*}

Distance en $\mathrm{km}$

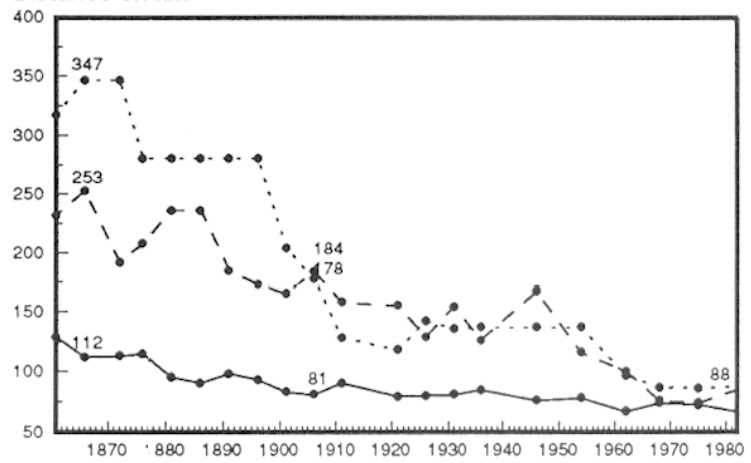

50000-100000 HAB. 100000-200000 HAB. 200000 HAB. ET PLUS

\section{II-B - Les petites villes*}

Distance en $\mathrm{km}$

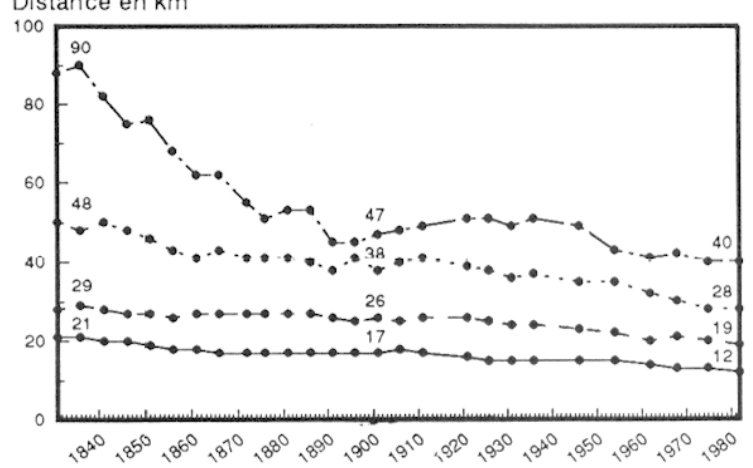

2000.5000 HAB. 5000-10000 HAB. 10000-20000 HAB. 20000-50000 HAB.

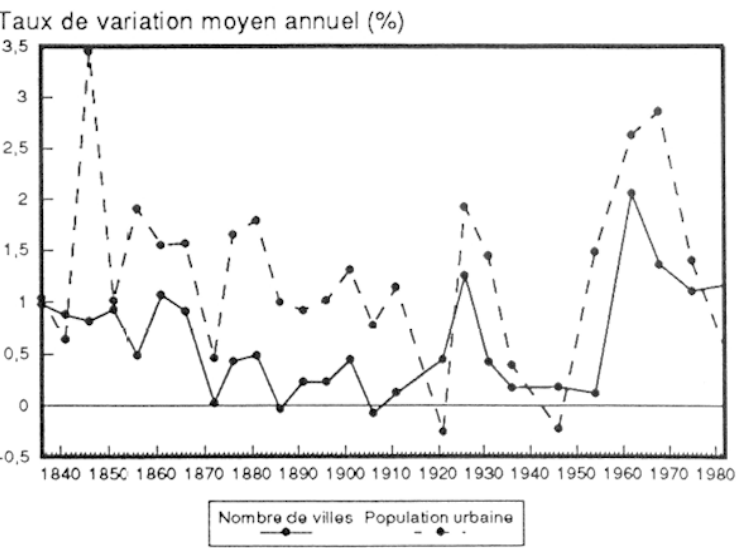

La croissance de la population urbaine française a été relativement lente pendant tout le $\mathrm{XIX}^{\mathrm{c}}$ siècle mais s'est accélérée au cours des quarante dernières années : on dénombrait 6 millions d'urbains en 1831, 15 millions en 1891, et 29 millions en 1954, on en compte 40 millions aujourd'hui. Le taux d'urbanisation français est resté longtemps en retard sur celui des autres pays d'Europe occidentale : $19 \%$ en $1831,36 \%$ en $1891,55 \%$ en 1954 , il atteint $73 \%$ en 1982 (en Grande-Bretagne, le taux d'urbanisation était de $44 \%$ dès 1831).

Ce processus d'urbanisation eut donc un démarrage tardif et fut longtemps modéré : la progression de la population urbaine n'a été que de $1 \%$ par an en moyenne au XIX ${ }^{e}$ siècle contre $2 \%$ en GrandeBretagne par exemple. Il n'a pris son essor qu'après la seconde guerre mondiale : $2,1 \%$ par an de 1946 à 1975 , pour atteindre assez rapidement, vers 1975 , sa phase de saturation.

\section{Des villes trois fois plus nombreuses et deux fois plus grandes}

Le nombre d'unités urbaines a augmenté : elles étaient 617 en 1831, elles sont 1781 en 1982. L'apparition de villes "nouvelles", qui sont en général des communes rurales ayant franchi le seuil de 2000 habitants, est toutefois moins rapide que l'accroissement du nombre des citadins (graphique I). Pendant que la population urbaine était multipliée par sept, le nombre de villes a seulement triplé et leur taille moyenne a doublé. Sur l'ensemble de la période, la population urbaine s'est accrue en moyenne de $1,2 \%$ chaque année, et le nombre de villes de $0,7 \%$. Dès lors, la distance moyenne entre les villes s'est trouvée considérablement réduite (graphique II).

Observés sur les intervalles courts qui séparent les recensements (de 5 à 10 ans), les taux de croissance de la population et du nombre des unités urbaines sont extrêmement fluctuants (graphique III) : en dehors des périodes incluant les guerres, ils sont toujours positifs (1). Ces évolutions ne dessinent pas de véritables cycles. Tout au plus observe-t-on une certaine alternance entre des périodes d'urbanisation plus intense, avec des taux qui dépassent $1,2 \%$ par an, par exemple en 1856-1861, 1872-1881, 1926-1931 et surtout 1946-1968, et des périodes où la croissance est nettement plus faible, avec des taux qui peuvent descendre jusqu'à $0,4 \%$ par an. Dans l'ensemble, taux élevés et taux faibles se succèdent de façon peu régulière, apparemment imprévisible. Mais il est absolument remarquable que ces importantes fluctuations de la population urbaine et du nombre des villes se produisent presque toujours dans les mêmes périodes. Le développement urbain est donc extrêmement cohérent ; il procède simultanément par accroissement de la population des villes et par création de nouvelles unités urbaines, à l'intérieur d'un processus d'urbanisation qui s'effectue par à-coups.

1. Le taux exceptionnellement élevé de la période 1841-1846 est un artefact dû̀ la mauvaise qualité du recensement de 1841 qui comporte des sous-enregistrements systématiques. 
* Les pourcentages expriment la part de la population urbaine totale qui habite dans une classe de taille de ville.

\section{Graphique IV \\ Les grandes villes ont un poids croissant*}

Population totale par classe de taille en $\%$

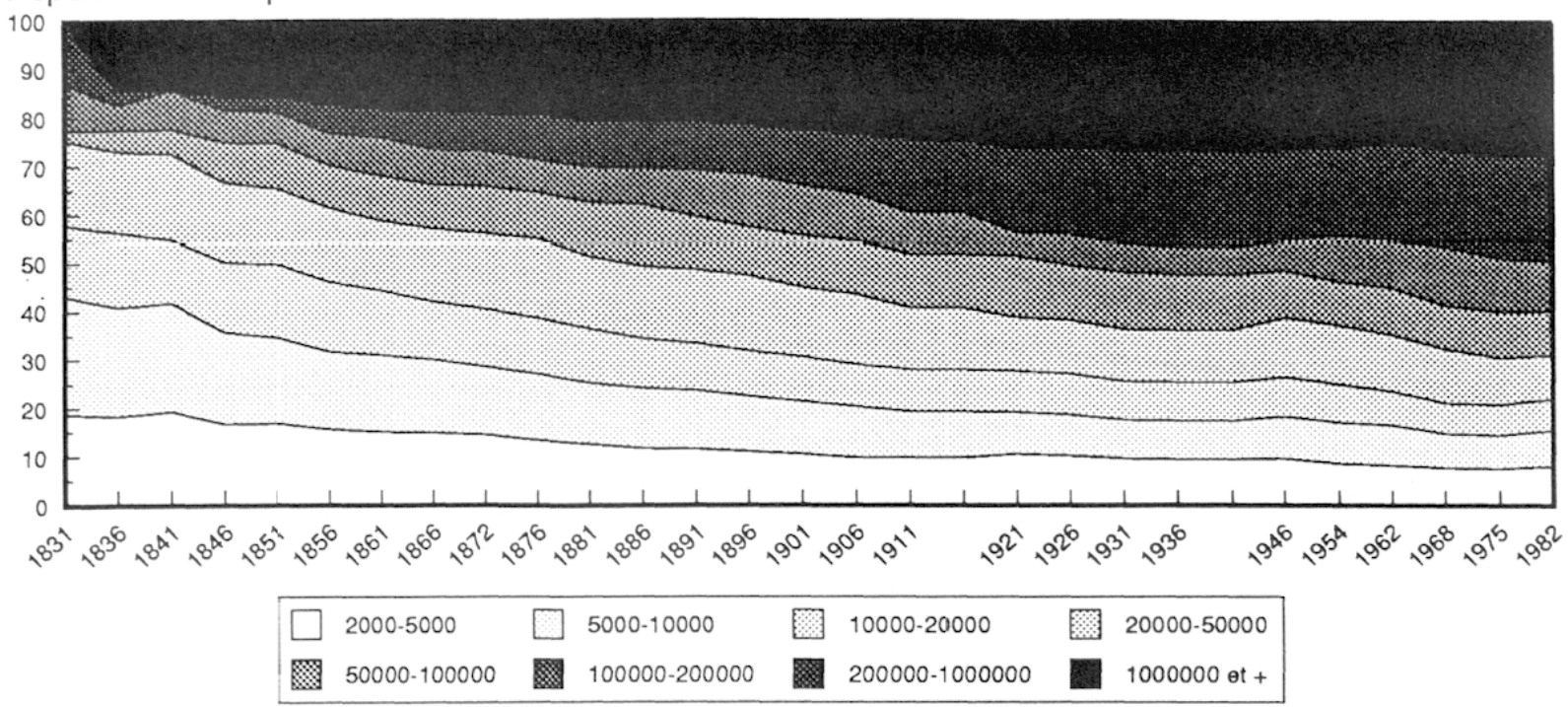

\section{Graphique V}

\section{Distribution rang-taille des unités urbaines françaises *}

\section{POPULATION}

(en milliers)

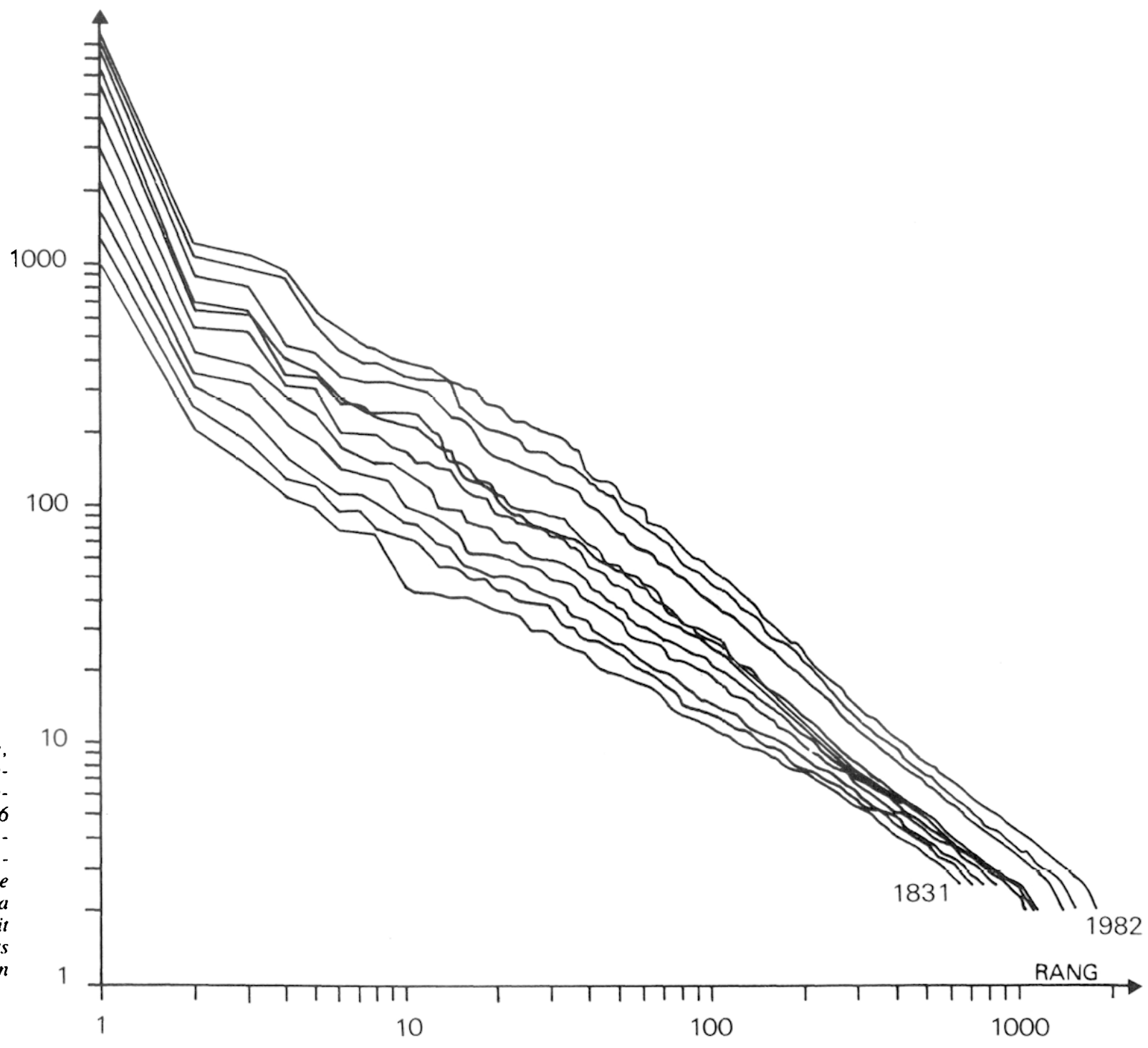

* De gauche à droite. les courbes correspondent aux dates suivan tes : $1831-1846-1856$ - $1872-1886-1906$ 1936 - 1946 - 1962. 1968 - 1982. L'échelle est logarithmique. La dixieme ville concentrait près de 40.000 habitants en 1831, et 130.000 en 1982. 
Au cours de ce siècle et demi d'urbanisation, la répartition de la population entre les différentes catégories de taille de villes s'est modifiée. En 1831 , près de la moitié de la population urbaine (43\%) habitait dans les petites villes (moins de 10.000 habitants), $35 \%$ dans les villes de taille moyenne et seulement $22 \%$ dans les villes de plus de 100.000 habitants; en 1982 , les proportions sont inversées : $15 \%$ des urbains résident dans les petites villes, $25 \%$ dans les villes de taille moyenne et $60 \%$ dans les plus grandes villes. Cette concentration de la population dans les grandes villes a été progressive. Elle s'explique par un accroissement de la taille moyenne des unités urbaines mais aussi par une croissance souvent plus rapide des grandes villes (graphique IV).

\section{La taille des villes : une hiérarchie persistante...}

Dans son ensemble, la forme de la distribution rangtaille est resté stable tout au long du siècle et demi écoulé (encadré ci-contre). Par rapport aux hiérarchies urbaines d'autres pays, elle est marquée par la primauté de la capitale : le rapport est de 1 à 7 entre la population parisienne et celle de la seconde ville, alors que le modèle de Zipf prévoit un rapport de 1 à 2 seulement (encadré ci-contre). La persistance dans le temps de cette primauté est remarquable : son origine remonte vraisemblablement à des périodes bien antérieures au $\mathrm{XIX}^{c}$ siècle. Les cent cinquante dernières années l'ont préservée sans l'accentuer sensiblement.

Les trente premières villes françaises ont maintenu entre elles des inégalités à peu près constantes, ce que traduit l'assez grand parallélisme des courbes rang-taille. Entre la $10^{e}$ et la $100^{e}$ ville, on observe cependant un changement bien perceptible dans l'allure des courbes, qui présentent en 1831 une forme concave et en 1982 une forme légèrement convexe. Durant cette période, le niveau intermédiaire de la hiérarchie des tailles de villes $(40.000$ à 450.000 habitants) s'est donc renforcé (graphique $\mathrm{V}$ ).

\section{...mais des inégalités de taille de plus en plus fortes}

En fait, globalement, les villes ont des tailles de plus en plus différentes. Le processus d'urbanisation s'est accompagné d'un mouvement de concentration de la population dans les plus grandes villes. Cette concentration est exprimée par l'augmentation régulière entre 1831 et 1982 , de la valeur absolue de la pente de la droite qui ajuste chaque distribution rang-taille (graphique VI). Ce processus de concentration, continu depuis le début du XIX $\mathrm{XIè}^{\mathrm{e}}$ cle, s'est ralenti ou stabilisé entre 1926 et 1954 . Il s'est accéléré entre 1954 et 1975 , durant la phase d'urbanisation la plus rapide de toute l'histoire des villes françaises. La tendance s'est nettement retournée depuis 1975 : désormais, la population des villes petites et moyennes augmente plus vite que celle des grandes.
La signification de cette tendance nouvelle à la déconcentration est discutée : faut-il y voir l'amorce d'un mouvement durable de redistribution de la population vers les villes moyennes et petites, qui tendrait à réduire les inégalités de taille des villes? Telle est la thèse de J.B.Parr [1], qui a obtenu pour plusieurs pays des "courbes en forme de $U$ " en calculant la valeur absolue de la pente de la distribution rang-taille pour des périodes allant de 1930 à 1980. En fait, les données utilisées par cet auteur introduisent un biais : son échantillon de villes est parfois limité à la partie supérieure de la hiérarchie

\section{Graphique VI \\ Des tailles de villes de plus en plus inégales*}

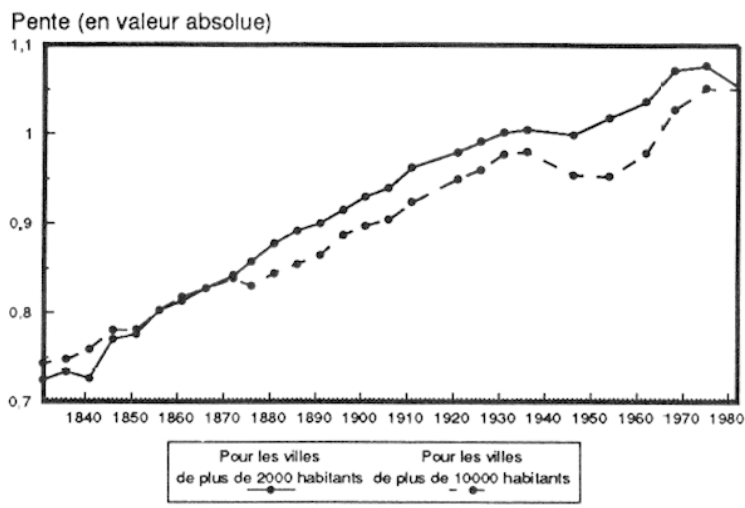

* Evolution de la pente de la droite ajustant la distribution rangtaille (en valeur absolue).

\section{Une distribution de Pareto}

Quelle que soit la période considérée, il existe beaucoup de petites villes et peu de grandes villes et le nombre des villes diminue selon une progression géométrique régulière lorsqu'on considère des catégories de taille de plus en plus élevée. La distribution de la taille des villes, très dissymétrique, ressemble à une loi lognormale ou à une distribution de Pareto. La régularité de cette organisation hiérarchique n'est pas propre à la France, et a été remarquée depuis très longtemps. La formulation peut-être la plus célébre en a été donnée par Zipf (en 1949), qui l'a dénommée "loi rang-taille" : si les villes d'un pays sont rangees d'après leur taille, de la plus grande à la plus petite, la population $P_{i}$ d'une ville est lié à son rang $r_{i}$ par la relation :

$r_{i} P_{i}^{q}=K$

( $K$ et $q$ étant des constantes)

On représente souvent cette relation sur un graphique à doubles coordonnées logarithmiques où la population des villes est en ordonnée et leur rang en abscisse. Les points s'alignent alors approximativement le long d'une droite de pente négative. La valeur absolue de cette pente est d'autant plus élevée que la taille des villes est dispersée. Nous avons représenté cette dis. tribution rang-taille pour l'ensemble des unités urbaines à chaque date et sélectionné les courbes qui permettaient de suivre le mieux l'évolution de la hiérarchie urbaine au cours du temps (graphiques $V$ et $V I$ ). 
urbaine, et il n'a mesuré les populations que dans le cadre des villes-centres et non dans la limite des agglomérations. La saturation des villes-centres des plus grandes zones urbaines explique alors pour une grande part la diminution de la valeur absolue de la pente de la distribution rang-taille. Dans ce cas, on peut d'ailleurs se demander si la diminution observée entre 1975 et 1982 ne s'explique pas tout simplement par l'ampleur de la péri-urbanisation, qui reporte une importante fraction de la croissance urbaine au-delà des limites des agglomérations, et cela d'autant plus qu'elles sont de grande taille.

On ne saurait donc négliger une autre interprétation selon laquelle le ralentissement de la concentration est seulement une oscillation de plus court terme, qui n'affecte pas sensiblement la forme de la hiérarchie urbaine. Toute l'histoire antérieure du processus d'urbanisation rend en effet très peu plausible l'hypothèse extrême de l'égalisation des populations des villes, qui a été avancée par certains analystes du "déclin" ou de la "fin des villes", voire prônée par les tenants d'une "taille optimale", des villes (Bairoch [2]).

\section{Un exemple de modèle explicatif}

La distribution de la taille des villes, par sa dissymétrie très accentuée, sa généralité d'un pays à l'autre,et son étonnante persistance au cours du temps, a intrigué bien des chercheurs, qui ont tenté de l'expliquer en recourant à de très nombreuses théories.

\section{Une explication : la loi de l'effet proportionnel}

Nous avons choisi de tester l'un des modèles qui cxpliquent la formation et le maintien d'une distribution très dissymétrique par des hypothèses relatives à la répartition de la croissance des villes. Le modèle le plus connu et le plus utilisé est celui de Gibrat (1931) [3]. Il montre qu'une distribution de taille de villes devient toujours lognormale si les villes croissent selon un processus particulier, qu'il appelle : "loi de l'effet proportionnel". Les conditions sont les suivantes :

- Sur de courts intervalles de temps, la population $P$ de chaque ville s'accroit de petites quantités de population $\mathrm{dP}$ qui sont proportionnelles à sa taille; - Les taux de variation $\mathrm{dP} / \mathrm{P}$ de la population des villes sont des variables aléatoires, non corrélées avec la taille des villes, et indépendantes d'un intervalle de temps à l'autre.

\section{Une croissance aléatoire des villes ?}

Quelle que soit la période considérée, la croissance de la population des villes est très variable d'une ville à l'autre. La distribution statistique de ces taux de croissance est proche d'une distribution normale, mais est assez fortement dispersée. Ainsi, alors que les minima sont fréquemment de l'ordre de $-6 \%$ par an, les maxima dépassent assez souvent $9 \%$, et en moyenne, une ville sur trois est en déclin. Les phases d'urbanisation intense sont celles qui engendrent le plus d'inégalités de croissance parmi les villes : les valeurs des écarts-types varient en phase avec celles de la moyenne. Cependant, depuis 1954, la dispersion relative des taux de croissance s'atténue : les valeurs de la moyenne des taux et de l'écart-type sont presque égales, alors qu'auparavant la valeur de l'écart-type représentait 2 à 3 fois celle du taux moyen (graphique VII).

Dans l'ensemble, la loi de l'effet proportionnel est donc une assez bonne approximation de ce processus de croissance : le nombre d'habitants qui s'ajoutent à la population de chaque ville entre deux recensements est à peu près proportionnel à l'importance de cette population. Les taux de croissance ont des corrélations faibles, quoique toujours positives, avec la taille des villes et sont peu corrélés entre eux d'une période sur l'autre. La loi de l'effet proportionnel permet donc de rendre compte de la persistance de la forme de la hiérarchie urbaine et de la concentration progressive de la population urbaine dans les plus grandes villes.

Pourquoi la croissance des villes vérifie-t-elle les deux hypothèses fondamentales de Gibrat ? Il existe des conditions générales plus ou moins favorables à la croissance des populations urbaines, comme la croissance démographique et économique, la substitution d'une économie industrielle et tertiaire à l'ancienne économie agricole provoquant un exode rural, ou encore, l'amélioration des conditions techniques qui permettent de faire fonctionner des villes de plus en plus grandes. Chaque ville subit ces forces d'urbanisation, qui se traduisent par une tendance au développement de même intensité, mais la diversité des conditions locales introduit des variations de la croissance d'une ville à l'autre. Les facteurs de la croissance d'une ville sont en effet très nombreux, d'origine très diverse, et sont souvent indépendants les uns des autres. A ces variations locales s'ajoutent des fluctuations dans le temps qui s'expliquent de la même façon.

\section{Graphique VII \\ Plus la croissance est forte, plus elle est inégale*}

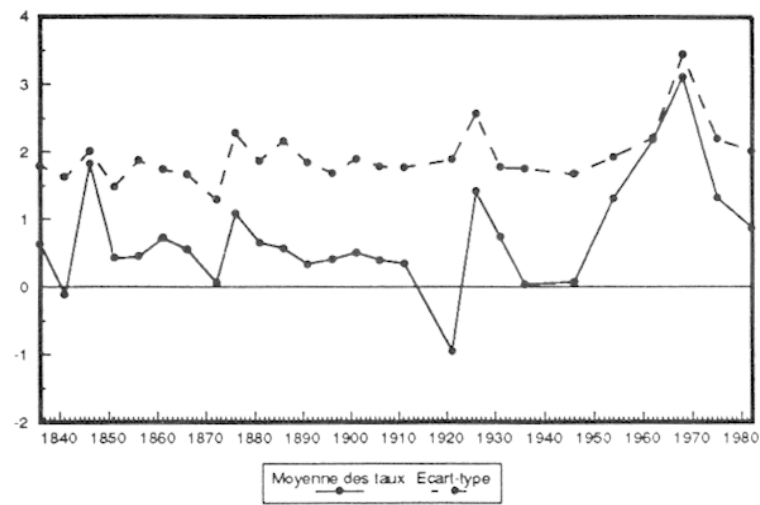

* L'écart-npe est un indicateur de dispersion. 
Il reste à expliquer pourquoi la croissance est proportionnelle à la taille des villes. Cette croissance a deux composantes : l'accroissement naturel et le solde migratoire. La croissance naturelle des populations, lorsqu'il n'y a pas de limites de ressources, est multiplicative, proportionnelle aux populations initiales. Le solde migratoire est lui aussi en moyenne proportionnel à la taille des villes, puisque le nombre de personnes entrées et sorties d'une ville est directement dépendant de la taille de celle-ci.

Toutefois, même faibles, les écarts au modèle sont trop systématiques et souvent assez significatifs. D'autres aspects du processus doivent donc être pris en compte.

\section{Croissance urbaine et diffusion des innovations}

Le premier type d'écart au modèle de Gibrat apparaît en France d'une manière épisodique: il s'agit de l'élévation des taux de croissance avec la taille des villes (graphique VIII). La corrélation d'ensemble entre les taux de croissance et la population est significative, même si les coefficients restent assez faibles sur toutc la pćriodc (proches de 0,05). Si l'on considère la corrélation entre les taux de croissance et le logarithme de la taille des villes, la valeur des coefficients s'élève à 0,3 entre 1831 et 1911 et diminue régulièrement à partir de 1954 pour devenir nulle en 1975-1982. S'il existe une relation entre le taux de croissance des villes et leur taille, elle est donc plus proche d'une relation de type logarithmique que d'une relation linéaire.

$\mathrm{Au} \mathrm{XIX}^{\mathrm{e}}$ siècle, cette corrélation entre croissance et taille des villes exprime surtout la faiblesse de la croissance des villes de moins de 10.000 habitants. Leurs taux de croissance sont en moyenne égaux, ou au plus deux fois supérieurs, à celui de la population française totale, alors que les villes de plus de 10.000 habitants ont crû en moyenne cinq fois plus vite. Après 1945, ce sont d'abord les plus grandes villes, jusqu'au seuil de 200.000 habitants, qui se sont développées le plus rapidement, en moyenne deux fois plus vite que les autres. Les taux de croissance se sont progressivement égalisés et depuis 1968 la tendance s'est inversée, ce sont les petites villes de moins de 10.000 habitants qui croissent désormais en moyenne trois fois plus vite que celles de plus de 100.000 habitants. Ce processus ne se poursuit-il pas aujourd'hui jusque parmi les communes rurales, avec une forte impulsion donnée aux plus grandes d'entre elles (entre 1000 et 2000 habitants)?

Cette interprétation se trouve renforcée par l'observation du deuxième type d'écart au modèle de Gibrat. Il s'agit de l'élévation de la corrélation entre les taux de croissance à deux intervalles de temps successifs (autocorrélation temporelle). Cette corrélation est particulièrement élevée pour les périodes 1846-1876, 1886-1911 et 1954-1982 (environ $0,4)$, où l'urbanisation fut très intense. Le processus d'urbanisation est donc sélectif puisque, durant ces longs intervalles de temps, ce sont les mêmes villes qui se développent, qui stagnent ou qui déclinent.

Il est tentant de relier ces deux caractéristiques du processus de croissance aux différentes vagues d'innovation économiques ou techniques qui se sont succédées dans le temps. Elles ont souvent favorisé pendant une période de temps plus ou moins longue un certain type de villes, que leur situation géographique ou socio-économique plaçait momentanément en position favorable. En général, les innovations apparaissent dans les plus grandes villes et se diffusent ensuite jusque dans les plus petites villes, selon ce que l'on appelle un processus de diffusion hiérarchique. Cette diffusion est facilitée par les nombreuses relations, de complémentarité ou de concurrence, qui existent dans un ensemble de villes, et qui ont amené les géographes à employer le concept de réseau urbain.

L'élévation du taux de croissance avec la taille des villes et l'autocorrélation temporelle des taux sont liées aux phases de croissance économique et urbaine les plus rapides. Ces corrélations accentuent

\section{Graphique VIII}

\section{Une croissance aléatoire?}

VIII-A - Corrélation entre les taux de variation de la population des villes pris à des périodes successives

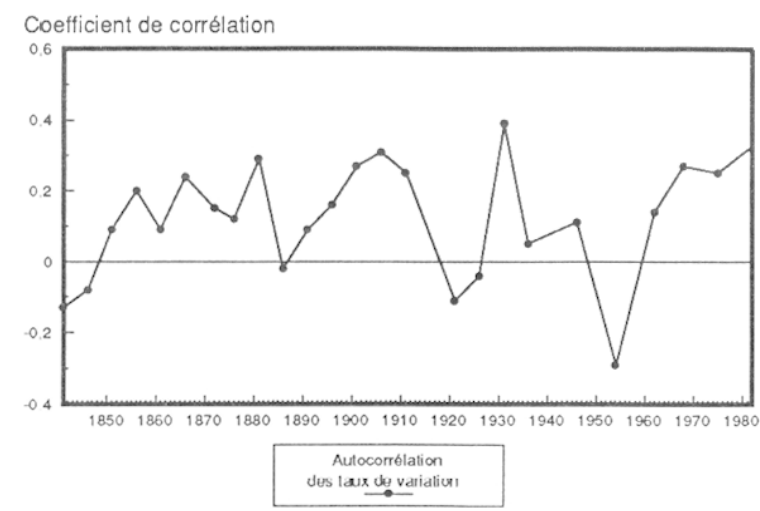

VIII-B - Corrélation entre les taux de variation de la population des villes et leur taille

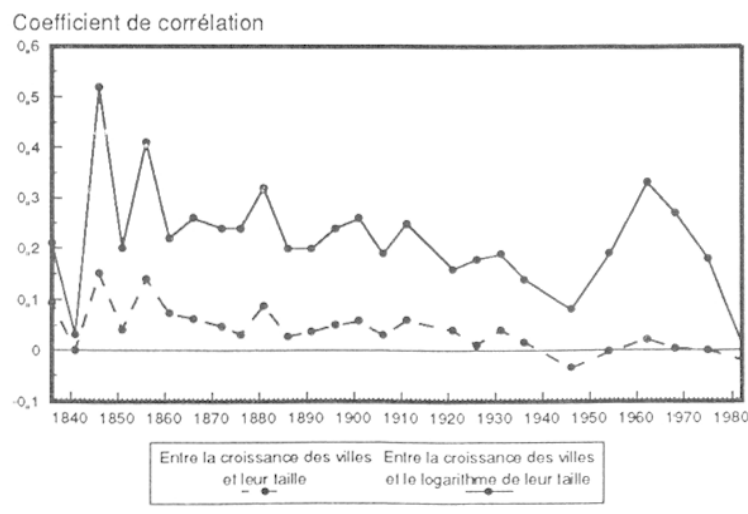


la concentration et la dissymétrie de la distribution des tailles de villes. Mais ces périodes de forte croissance urbaine n'ont pas une intensité et une durée suffisantes pour perturber le fonctionnement du processus majeur de croissance obéissant à la loi de l'effet proportionnel.

Finalement, l'aspect aléatoire du processus de croissance, qui se traduit sur la longue durée par un développement très cohérent de l'ensemble des villes, s'explique par une diffusion trc̀s poussée des innovations de toute nature dans toutes les villes, même les plus petites. Les décalages introduits par le fait que cette diffusion emprunte la voie hiérarchique dans le réseau urbain, rendent compte des écarts au modèle stochastique de Gibrat. L'observation de la répartition spatiale de la croissance amène cependant à compléter cette interprétation en incorporant un autre facteur de variation qui est la dimension géographique du système des villes.

Géographie de la croissance urbaine

La croissance ou le déclin urbain ne sont jamais limités à une seule région pour une période déterminée (cartes 2 à 4). La règle est au contraire une très grande dispersion de la croissance urbaine sur tout le territoire, avec une répartition géographique le plus souvent quasi-aléatoire (semis de Poisson) (Guérin-Pace, [4]). Il est cependant possible d'identifier des zones qui ont été un peu plus favorisées, notamment par la diffusion restreinte de formes de développement économique assez concentrées : villes minières et sidérurgiques du nord et de l'est, mais aussi villes de la Côte d' $\Lambda$ zur au XIX ${ }^{\mathrm{e}}$ siècle, et générations des villes développées grâce à l'électricité de la zone alpine dans l'entre deuxguerres. Signalons encore le centre et les marges occidentales et méridionales du Bassin parisien (industrialisés entre autres par la décentralisation et la diffusion de l'électronique), mais aussi le développement diversifié des villes de la région lyonnaise et alpine et le développement touristicotertiaire de la zone méditerranéenne au cours des trente dernières années.

Cependant, à partir de 1954, la croissance de la population des villes devient plus systématiquement concentrée géographiquement. On distingue alors des zones où se groupent les villes en forte croissance, telles la couronne parisienne et l'axe rhodanien, et des ensembles de villes en déclin, surtout dans les vieilles régions industrielles du Nord et de la Lorraine. Ces vagues de diffusion régionale de la croissance n'épuisent cependant pas toute la dimension géographique de l'évolution des réseaux de villes.

\section{Carte 2}

Taux de variations moyens annuels de 1831 à 1911

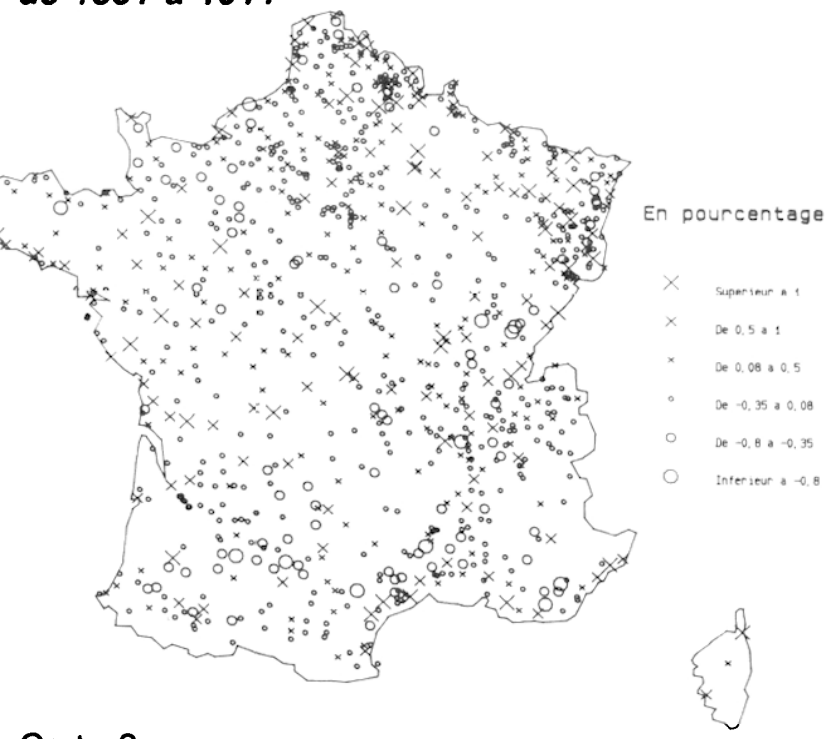

\section{Carte 3}

Taux de variations moyens annuels de 1911 à 1954

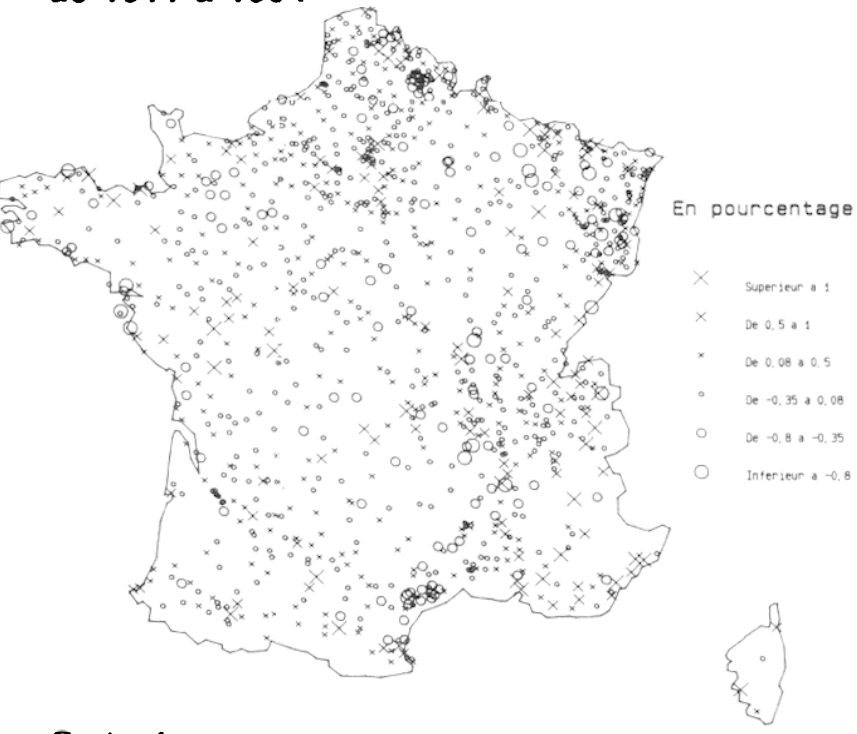

\section{Carte 4}

Taux de variations moyens annuels de 1954 à 1982

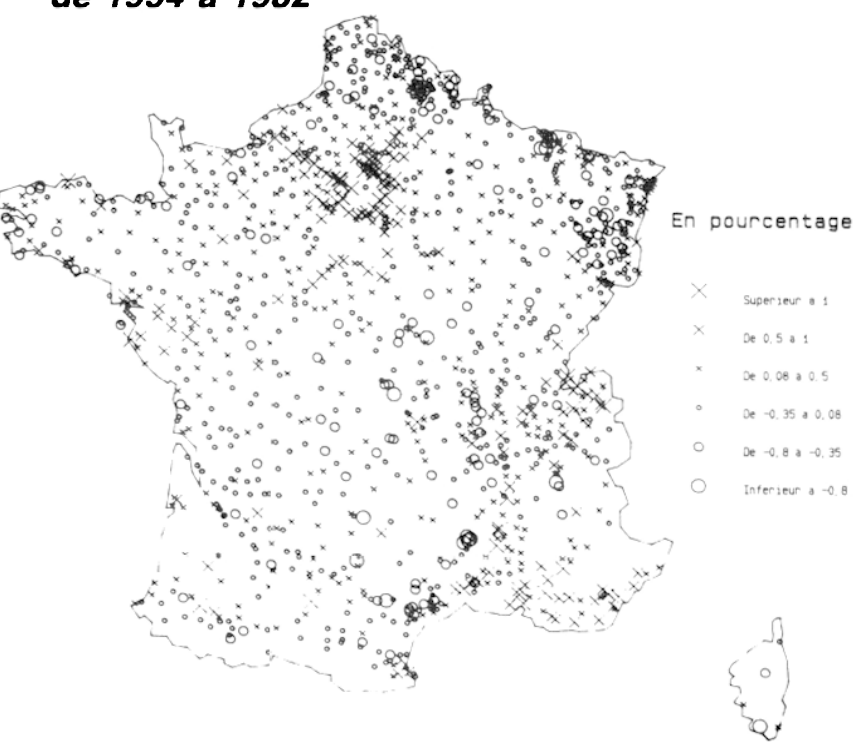




\section{La compétition entre les villes}

Une méthode classique de mise en évidence des croissances différentielles considère l'évolution du rang de chaque ville dans la hiérarchie urbaine. Le graphique IX illustre les changements de rang, entre 1831 et 1982 , des cent premières villes françaises de 1831. Quelle que soit la position initiale d'une ville dans la hiérarchie, on observe aussi bien des gains que des pertes de rang. Ainsi, la compétition se joue entre les villes de tout rang, c'est un processus caractéristique du système urbain à tous les niveaux. Toulon, Nice, Grenoble ou Valenciennes ont été gagnantes, mais aussi La Rochelle, Pau et Saint-Chamond, tandis qu'au contraire, Amiens, Troyes, Montauban, Abbeville et Issoudun dégringolaient dans la hiérarchie. Plus l'on descend vers le bas de cette hiérarchie, plus l'amplitude des mouvements gains-pertes devient importante. Cela signifie que les villes de petite taille perdent ou gagnent plus facilement un grand nombre de rangs que des villes situées dans la partie supérieure de la hiérarchie, qui apparaît comme étant plus stable. La statistique des changements de rang ne permet pas cependant de comparer directement des villes de taille différente, puisque la probabilité de gagner ou de perdre un rang correspond à des variations relatives de population plus élevées pour les grandes villes que pour les petites.

\section{Des villes champignons aux villes sans avenir}

Une méthode plus synthétique pour décrire les différences de croissance consiste à ordonner les courbes qui retracent l'évolution des populations de chaque ville de 1831 à 1982 , au moyen d'une classification ascendante hiérarchique. On obtient ainsi des types de trajectoires qui caractérisent chacune une évolution particulière de certaines villes dans le réseau urbain. L'interprétation des types de cour- bes obtenues est ensuite facilitée si l'on rapporte à chaque date la population des villes considérées à la population urbaine totale. On fait ainsi abstraction de la croissance du système urbain, en ne considérant que l'évolution relative de la population des villes (graphique $X$ ).

On peut distinguer six principaux types d'évolution de la population, répartis en deux groupes. Le premier groupe rassemble quatre types de villes, qui ont en commun d'avoir connu à un moment ou à un autre, au cours du siècle et demi écoulé, une croissance relative plus rapide que celle de la moyenne des villes.

Le type 1 concerne des villes qui ont crû très rapidement tout au long du XIX ${ }^{e}$ siècle et qui ont encore accéléré leur croissance depuis 1936. Ce sont essentiellement des villes du Sud-Est, telles Cannes, Nice, Grenoble ainsi que quelques "villeschampignons" liées aux croissances très rapides de la première révolution industrielle ou de la période récente, de la région du Nord (Lens, Valenciennes, Béthune) et de la région parisienne (Mantes-la-Jolie, Melun). Ces villes avaient des dimensions modestes à l'origine. Elles ont considérablement amélioré leur position dans la hiérarchie urbaine.

Les villes du type 2 se concentrent essentiellement dans deux régions, le couloir rhodanien et une couronne de villes de taille moyenne autour de Paris, avec des prolongements vers le sud-ouest du bassin parisien. Toutes ont eu une croissance très moyenne ou parfois un déclin relatif de leur population tout au long du $\mathrm{XIX}^{\mathrm{e}}$ siècle, suivi d'une reprise de croissance depuis les quarante dernières années. Toulouse et Lille sont des métropoles régionales qui ont connu une évolution semblable, ainsi que Toulon, Montpellier, Clermont-Ferrand, Dijon, Orléans et Tours.

Les deux types suivants ont connu une période de croissance assez rapide, suivie d'une stagnation ou
* Sur l'axe horizontal figure la position initiale des 100 premières villes en 1831. Sur l'axe vertical est reportée la variation de cette position par rapport à celle atteinte en 1982. Les trois premières villes françaises sont les mêmes depuis un siècle et demi. Mais Rouen. qui était la sixième ville de France en 1831 , n'est plus dans les dix premières en 1982. Les villes qui ont gagné des places sont au dessus de la ligne horizontale (TouIon, Nice, Grenoble...). Les changements de rangs sont d'autant plus importants que la position initiale était éloigné dans la hiérarchie. Issoudun, par exemple. érait la $89^{\circ}$ ville en 1831 mais a perdu plus de 200 places depuis.

\section{Graphique IX Compétition dans la hiérarchie urbaine*}

\section{Variations du rang entre 1831 et 1982}

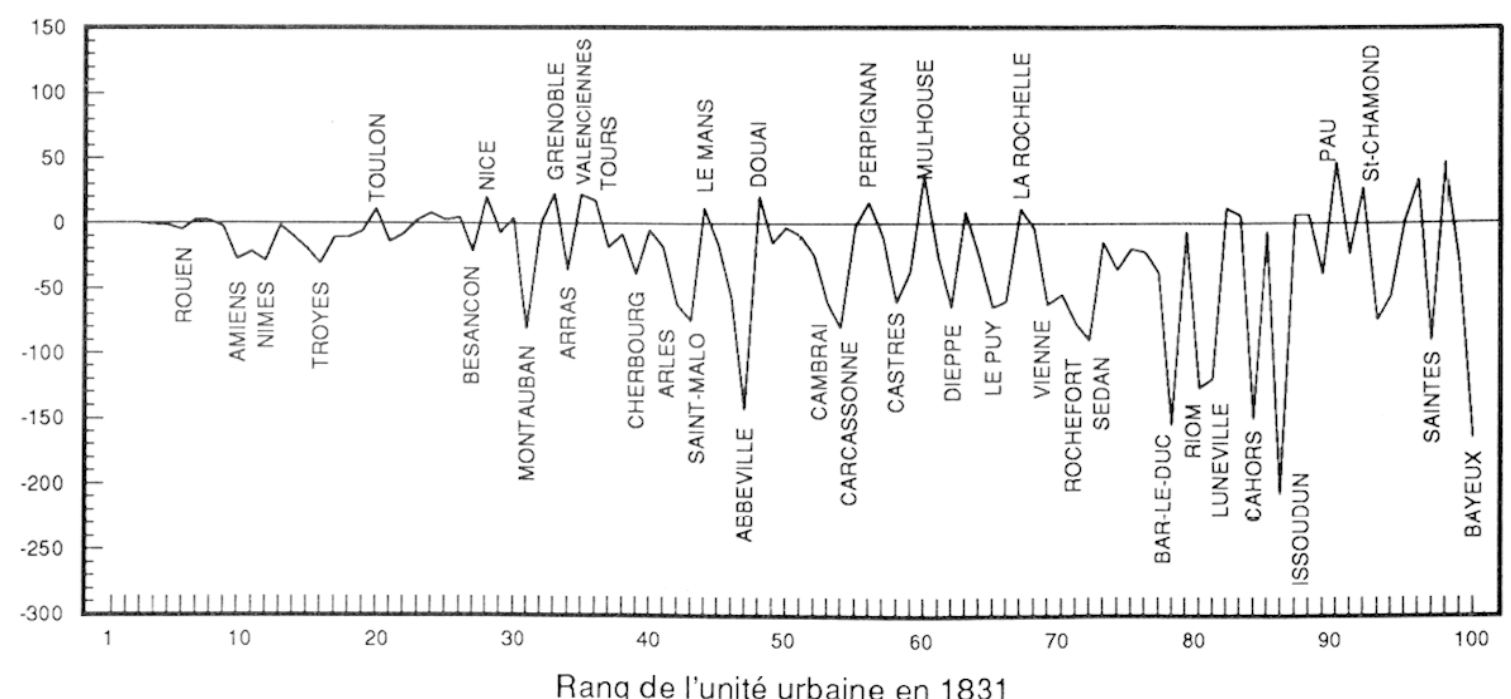




\section{$X-A$ - Population moyenne}

Pop moyenne $(\log )$

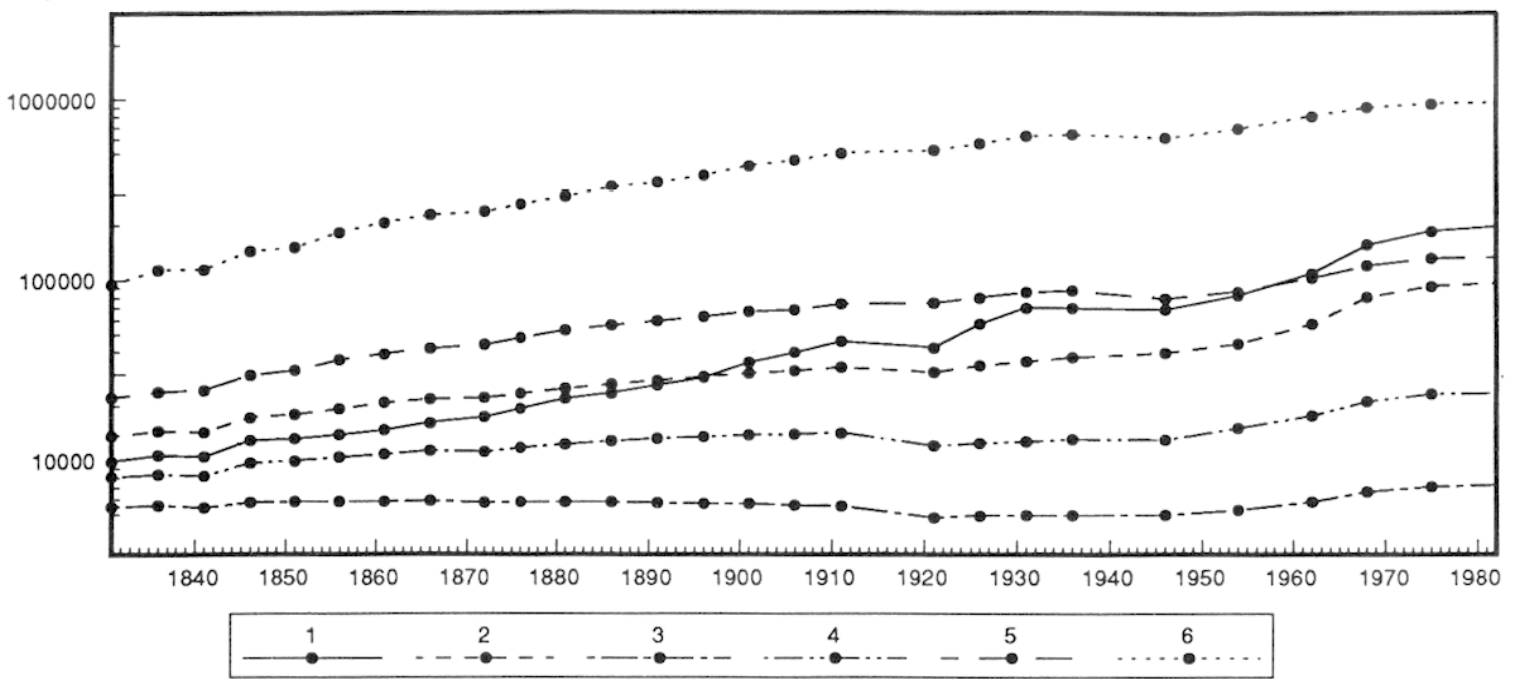

\section{$X-B$ - Poids relatif dans l'ensemble des villes}

Importance relative des villes

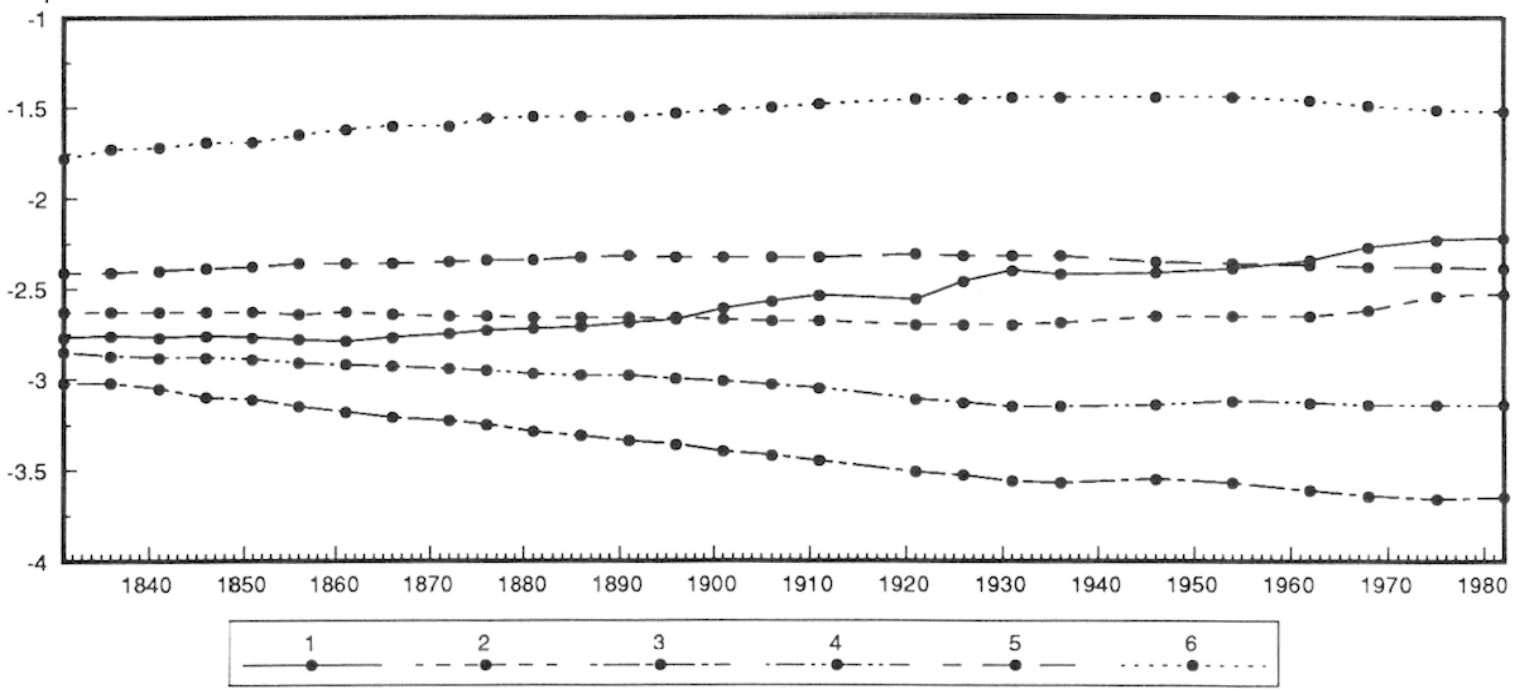

* Dans ces deux graphiques, les valeurs sont indiquées en logarithme ce qui permet de tasser des évolutions parfois explosives. Le premier graphique représente l'évolution de la population movenne des six types de villes. Le second, celle de leur part relative. Cette part est calculée de la manière suivante : au numérateur figure la population moyenne d'un des types de villes; au dénominateur figure la population urbaine totale à chaque recensement diminuée de la population de ce type de ville.

d'un déclin relatifs, qui se traduit par un maintien de leur position dans le système urbain.

Le type 3 rassemble des villes qui ont connu une croissance soutenue jusqu'en 1930 puis une saturation et une perte d'importance depuis 1960 . Ces villes telles Paris et Nancy sont peu nombreuses, mais souvent de grande taille.

Le type 4 est caractérisé par une croissance moins soutenue tout au long du XIX $\mathrm{X}^{\mathrm{e}}$ siècle, et un léger déclin à partir des années 1930. La plupart des très grandes villes appartiennent à ce groupe, comme Lyon, Marseille, Strasbourg, Nantes, Bordeaux, Mulhouse. Ces métropoles, bénéficiant des effets des cycles d'innovation successifs, ont réussi tout au long de cette période à maintenir leur place dans la hiérarchie urbaine.

Un deuxième groupe est composé des unités urbaines qui ont perdu de leur importance relative. Elles se distinguent de la façon suivante.

Le type 5 est constitué de villes de taille moyenne et petite (aucune n'atteint aujourd'hui 200.000 habitants), réparties dans toute la France. Elles ont subi tout au long du XIXe siècle une croissance très lente, qui s'est traduite par un déclin relatif. Une très légère reprise dans les années 1960 ne les a pas empêchées de continuer à perdre encore de leur 
importance dans la hiérarchie urbaine au cours des dernières années. Cette évolution laisse-t-elle à penser que le déclin urbain n'est jamais irréversible, ou bien ne s'agit-il que d'un sursis pour des centres condamnés au déclin du fait de leur petite taille?

Le dernier type d'évolution (type 6) tendrait à renforcer la seconde hypothèse. Il rassemble des villes qui, bien qu'elles aient un peu accru leur population, ont été en déclin relatif sur toute la période. Ce sont uniquement des petites villes (moins de 20.000 habitants en 1982, à l'exception de trois d'entre elles) et elles sont réparties sur tout le territoire français sans localisation privilégiée. Ces points faibles de la trame urbaine s'intercalent dans toutes les hiérarchies régionales.

\section{L'effet de la contraction espace-temps}

Cette disposition peut être interprétée comme révélant l'adaptation progressive du système urbain à des conditions socio-économiques et techniques changeantes. La croissance généralisée de la population des villes et l'accroissement de leur nombre répondent à la multiplication et à la diversification des fonctions urbaines liées à la division croissante du travail et à l'èlévation du niveau de la production et des consommations. Au cours de ce processus, la hiérarchie urbaine se développe dans son ensemble. Le passage des grands cycles d'innovation se traduit parfois par un privilège momentané de croissance pour certains types de ville, associés par exemple au développement des concentrations industrielles, que l'on décrit en termes de "générations de villes". Mais, dans le même temps, les avantages relatifs des grandes villes se renforcent par rapport à ceux des petites, en particulier du fait des conditions techniques de déplacement qui provoquent une contraction espace-temps sur le territoire. L'amélioration généralisée des moyens de transport favorise l'extension de l'aire d'influence des plus grandes villes qui concurrencent victorieusement le rayonnement des plus petites. Celles-ci se trouvent en quelque sorte "court-circuitées" par les grandes, même pour des fonctions assez élémentaires. En dépit du processus d'urbanisation qui gonfle en valeur absolue la population des petites villes, celles-ci perdent donc de leur importance relative dans le système, et cela quelle que soit la région considérée. Il s'ensuit que la hiérarchie urbaine, en même temps qu'elle se développe, se simplifie par le bas, c'est-à-dire par diminution du poids relatif de ses niveaux inférieurs.

Peu d'analyses de l'évolution de larges échantillons de villes ont été conduites sur des périodes aussi longues. Il serait souhaitable de pouvoir comparer ces résultats avec ceux d'autres pays afin de contrôler si ces effets de cohérence, de synergie dans la croissance des ensembles de villes, ont assez de généralité pour constituer une théorie de l'évolution des réseaux urbains. Des analogies existent entre l'évolution des villes et celles d'autres systèmes ouverts susceptibles d'auto-organisation (Pumain, Sanders, Saint-Julien, [5]), mais les systèmes urbains ont en outre une dynamique spécifique, liée à leur propriété d'objets à la fois historiques et géographiques.

\section{BIBLIOGRAPHIE}

[1] J.B. PARR : "A Note on the Size Distribution of Cities over Time", Journal of Urban Economics, vol 18, p. 195-212, 1985.

[2] P. BAIROCH : Taille des villes, conditions de vie et développement économique, EHESS, Paris, 1978.

[3] R. GIBRAT : Les inégalités économiques, Sirey, Paris, 1931.

[4] F. GUERIN-PACE : La dynamique d'un système de peuplement : évolution de la population des villes françaises de 1831 à 1982, Université Paris VII, Thèse de doctorat, 1990.

[5] D. PUMAIN, L. SANDERS, T. SAINTJULIEN : Villes et auto-organisation, Economica, Paris, 1989.
[6] D. PUMAIN : La dynamique des villes, Economica, Paris, 1982.

[7] D. PUMAIN, B. RIANDEY : "Le fichier de l'INED : Urbanisation de la France", Espace Populations Sociétés, n², p.269-278, 1986.

[8] INED : L'urbanisation de la France de 1831 à 1982, base de données informatisée, 1985.

[9] G. DUPEUX : Atlas historique de l'urbanisation de la France, CNRS, Paris, 1981.

[10] S. CHASSAGNE : "L'histoire des villes, une opération de rénovation historiographique", Villes et campagnes XVème-XXème siècle, C.H.E.S, P.U.L, Lyon, p.217-300, 1977. 